\title{
Transitional change in interaction between HIF-1 and HNF-4 in response to hypoxia
}

\begin{abstract}
The roles of the erythropoietin (Epo) 3' enhancer in the activation of gene expression in response to hypoxia were investigated. The enhancer contains hypoxiainducible enhancer binding site 1(HIF-1 element) and two direct repeats of hexanucleotide consensus nuclear receptor half site (HNF-4 element). HIF-1, which is a heterodimeric complex of HIF-1 $\alpha$ and aryl hydrocarbon receptor nuclear translecator (ARNT), binds to HIF-1 element. HNF-4 binds to HNF-4 element as a homodimeric complex. Studies on mutant reporter plasmids demonstrated that both HIF$1 \alpha$ and HNF-4 elements were necessary for augmentation of the enhancer activity, since mutation of either the HIF-1 or the HNF-4 element caused loss of inducibility under hypoxic conditions. Mammalian two-hybrid experiments in vivo revealed that transitional change took place from the interaction of HNF-4 with ARNT to that with HIF- $1 \alpha$ in response to hypoxia. Such interactive domains were identified in amino acids 369-465 containing the C-terminal of HNF-4 and amino acids 1-458 containing basic helix-loophelix (bHLH) and Per-ARNT-AHR-Sim (PAS) domains of ARNT in normoxia. Also, an extended sequence containing ligand and dimerization domains, and the C-terminal of HNF-4 (amino acids 135-465), and the PAS domain (amino acids 106-526) of HIF-1 $\alpha$ were used for the interaction between the two transcription factors in hypoxia. From these data, the functional significance of the transitional change in the augmentation of gene expression by the Epo enhancer in hypoxia is discussed.
\end{abstract}

Key words Erythropoietin · Enhancer · Hypoxia · HIF-1 · HNF-4 $\cdot$ Mammalian two-hybrid reporter assay

W. Zhang · T. Tsuchiya $\cdot$ Y. Yasukochi $(\bowtie)$

Department of Molecular Genetics, Medical Research Institute, Tokyo Medical and Dental University, 1-5-45 Yushima, Bunkyo-ku, Tokyo 113-8510, Japan

Tel. +81-3-5803-5825; Fax +81-3-5803-0248

e-mail: yyasmgen@mri.tmd.ac.jp

\section{Introduction}

Erythropoietin (Epo) is a protein hormone (Miyake et al. 1977), produced primarily in the kidneys and liver, that compensates reduced oxygen supply to organs by modulating erythropoiesis (Schuster et al. 1989; Bondurant and Koury 1986; Beru et al. 1986; Koury and Bondurant 1990). Transcription is a major control point in the augmentation of Epo gene expression. The best characterized model of regulated Epo expression is the human hepatoma cell line, Hep3B (Goldberg et al. 1987). These cells produce Epo in response to hypoxia and cobalt exposure by increasing the rate of transcription of the Epo gene.

The transcriptional response to lowered oxygen levels is mostly mediated by the enhancer region, which starts at 116bp downstream from the poly A addition site (Semenza and Wang 1992). This region is 50-nt in length, and contains two DNA elements. The first element is the hypoxiainducible enhancer binding site 1 (HIF-1 element), the highly conserved sequence near the $5^{\prime}$ end of the enhancer. This element is found in most of the genes that are up-regulated by hypoxia (Semenza et al. 1994; Levy et al. 1995; Ebert et al. 1995). The key step in their activation by hypoxia is considered to be the formation of the HIF-1 protein complex on the HIF-1 element (Wang and Semenza 1995; Wang et al. 1995). The HIF-1 complex is a heterodimeric complex of two basic helix-loop-helix Per-ARNT [aryl hydrocarbon receptor nuclear translator] AHR-Sim (PAS) proteins (Wang et al. 1995); ARNT, constitutively expressed, and HIF-1 $\alpha$, rapidly degraded under normoxic conditions by the ubiquitinproteasome system (Salceda and Caro 1997). Hypoxia induces stabilization of the HIF-1 $\alpha$, allowing the formation of the transcriptionally active complex.

The second element is two direct repeats of the hexanucleotide consensus nuclear receptor half site (HNF4 element) in the $3^{\prime}$ segment of the enhancer, which is highly conserved (Blanchard et al. 1992). Binding of hepatocyte nuclear factor 4 (HNF-4) to the HNF-4 element appears to increase the hypoxia-inducibility of Epo, since transient transfection of the reporter plasmid along with 
increasing amounts of HNF-4 results in a marked enhancement of luciferase production in hypoxic Hela cells (Galson et al. 1995). Although the individual effects of these two transcription factors on Epo enhancer activity were investigated, little experimental evidence is available about the nature of their cooperative role in the hypoxic induction of gene expression.

The present studies revealed that both HIF-1 and HNF4 elements were necessary for the augmentation of Epo enhancer activity in response to hypoxia. This synergistic up-regulation may result from transitional change in the interaction between HIF-1 and HNF-4 in hypoxia. In addition, their interaction domains were identified by each series of deletion mutants of HIF-1 $\alpha$, ARNT, and HNF-4.

\section{Materials and methods}

Cell culture

Hep3B cells were obtained from the American Type Culture Collection. The cells were grown in Dulbecco's modified eagle medium (D-MEM) containing $10 \%$ calf serum and $0.1 \mathrm{~g} / \mathrm{l}$ kanamycin in $95 \%$ air with $5 \% \mathrm{CO}_{2}$ (normoxia).
A hypoxic condition was generated by exposing the cells to $2 \% \mathrm{O}_{2}, 93 \% \mathrm{~N}_{2}$ and $5 \% \mathrm{CO}_{2}$.

\section{Plasmid constructs}

Plasmids for reporter assay of the enhancer activity of the Epo gene were constructed as follows. The human Epo promoter region $(0.2 \mathrm{~kb}$, Apa I-Eco52 I) was amplified by PCR with a pair of primers containing SacI and HindIII sites at the $5^{\prime}$ and $3^{\prime}$ ends, respectively, and inserted upstream of the luciferase cDNA of pGL2-basic vector (Promega, Madison, WI, USA). The human Epo enhancer region $(0.12 \mathrm{~kb}$, Apa I-Pvu II) was amplified by PCR with a pair of primers containing SalI sites at both ends, and cloned downstream of the poly (A) signal of the above construct, which was referred to as wild type. Mutants 1,2, and 3 in the enhancer element (Fig. 1) were constructed by the same method as the wild-type by using PCR products amplified with primers containing a 6-nt deletion in the HIF-1 element (M1), two 6-nt deletions in the HNF-4 element (M2), and a 6-nt and two 6-nt deletions in both the HIF-1 and HNF-4 elements (M3), respectively, as shown by $X X X X X X$ in Fig. 2.

Effector plasmids for the mammalian two-hybrid system in vivo were constructed as follows. The full length open
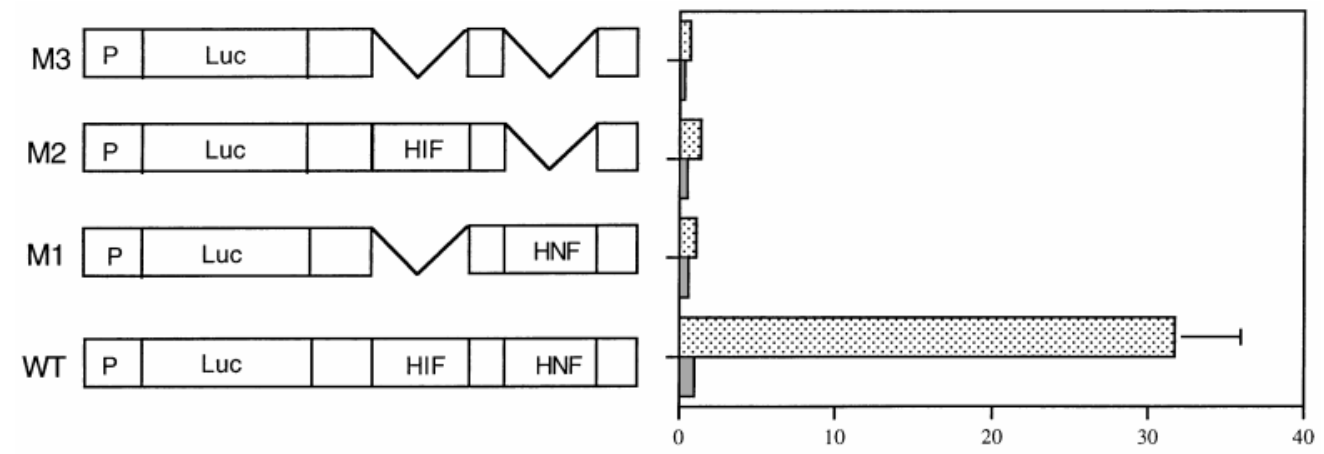

Relative luciferase expression

Fig. 1. Transient luciferase reporter assay by wild-type and mutant Epo enhancers in normoxia and hypoxia. Hep 3B cells were transfected with reporter plasmids, into which the wild-type Epo enhancer $(W T)$, or a mutant with a 6-nt deletion in the HIF-1 element (M1), or a mutant with two 6-nt deletions in the HNF-4 element (M2), or a mutant with a 6-nt deletion and two 6-nt deletions in both the HIF-1 and HNF-4 elements (M3), respectively, was cloned, and cultured in $20 \%$ oxygen (normoxia; gray bar) or in $2 \%$ oxygen (hypoxia; dotted bar) for $24 \mathrm{~h}$.
The cell extracts were assayed for luciferase activity by the method described in "Materials and methods." The relative luciferase activity was expressed as the ratio of the activity of reporter plasmid containing the wild-type enhancer in normoxia to the activities in the other experiments. Each bar represents the mean \pm SD of three independent experiments. $P$, Epo promoter; Luc, luciferase cDNA; HIF1, HIF-1 element; HNF4, HNF-4 element
Fig. 2. Enhancer element of the human erythropoietin (Epo) gene. The hypoxia-inducible enhancer binding site 1 (HIF-1 element) and two direct repeats of the hexanucleotide consensus nuclear receptor half site (HNF-4 element) are underlined. Number +121 indicates downstream from the poly A addition site of the Epo gene. A 6-nt deletion in the HIF-1 element and two 6-nt deletions in the HNF-4 element are shown by $X X X X X X$

\section{$+121$ IACGTGCTGTCTCACACAGCCTGTCTGACCTCTCGACCT $X \times X \times X$ $X \times X \times X X \quad X \times X X X$ \\ HIF-1 element \\ HNF-4 element}


reading frames of HNF-4, ARNT, and HIF- $1 \alpha$ were amplified by PCR with primers containing the BamHI site and NotI site with a stop codon at the $5^{\prime}$ and $3^{\prime}$ ends, respectively. After the resultant products were temporally cloned into pGEM-T vector (Promega), they were transferred to the NotI and BamHI sites of pBIND or pACT vector (Promega). Mutant effector plasmids for deletion experiments in the mammalian two-hybrid system were constructed by the same method as the effector plasmids described above, except that primers used for the amplification were between amino acid residues, 1-141, 142-458, 459-789, 1-458, and 142-789 of ARNT; 1-105, 106-526, $527-826,1-526$, and 106-826 of HIF-1 $\alpha$; and 1-134, 135$368,369-465,1-368$, and $135-465$ of HNF-4. All constructs were confirmed to be in frame by DNA sequencing.

\section{Reporter assay}

Hep3B cells $\left(2 \times 10^{5}\right.$ cells $)$ were seeded in a $60-\mathrm{mm}$ plate and cultured in $5 \mathrm{ml}$ of the growth medium supplemented with $10 \%$ calf serum until the cells reached $60 \%$ confluence. For each transfection, $1 \mu \mathrm{g}$ reporter plasmid in $100 \mu \mathrm{l}$ OPTIMEM I (GIBCO-BRL, Grand Island, NY, USA) was mixed with $5 \mu \mathrm{l}$ lipofection (GIBCO-BRL) in $100 \mu \mathrm{l}$ OPTIMEM I. Transfection was carried out by the addition of the mixture to the cells which had been washed twice with $2 \mathrm{ml}$ serum-free OPTI-MEM I, and incubation for $6 \mathrm{~h}$ in $95 \%$ air and $5 \% \mathrm{CO}_{2}$ at $37^{\circ} \mathrm{C}$. After transfection, the culture medium was replaced by $5 \mathrm{ml}$ D-MEM supplemented with $10 \%$ calf serum, and incubated for $15.5 \mathrm{~h}$ in normoxia, after which the cells continued to grow for up to $24 \mathrm{~h}$ in either the normoxic or hypoxic condition. At the end of the 24-h incubation after transfection, the cells were washed with phosphate-buffered saline (PBS) and lyzed with $500 \mu$ l cell lysis buffer (Toyo Ink, Tokyo, Japan). The luciferase activity of the cell lysate was measured for $1 \mathrm{~min}$ with a luminometer (Lumat LB 9501; Berthold, Bad Wildbad, Germany), using a luciferase assay system (Toyo Ink, Tokyo, Japan), and expressed as relative light units (RLUs). Gene transfer efficiency was normalized by $\beta$-galactosidase activity of pRSV- $\beta$-Gal used as an internal control.

The reporter assay for the mammalian two-hybrid system was carried out by the same method as above, except that $0.4 \mu \mathrm{g}$ each of the reporter plasmid (pG5luc, Promega) and two effector plasmids in $100 \mu$ OPTI-MEM I was mixed with $5 \mu \mathrm{l}$ lipofectin in $100 \mu \mathrm{l}$ OPTI-MEM I and were cotransfected either individually or in various combinations.

\section{Results}

Synergistic up-regulation of gene expression under hypoxic conditions by HIF-1 and HNF-4 elements in reporter assay

Previous studies have shown that a 50-nt element, which starts at 116bp downstream from the Epo gene polyadenylation site, functions as a minimal hypoxia-inducible enhancer in
Hep3B cells. Semenza and Wang (1992) dissected the element to three distinct regions including HIF-1 and HNF-4 elements, and demonstrated that, under hypoxic conditions, the deletion mutation in the HNF-4 element (nt 34-50 deletion) causes a decrease of inducibility by more than half, and of relative CAT expression by one fourth when compared with the 50-nt element, but the 3-nt substitution in the HIF-1 element causes almost no inducibility. However, the enhancer structure of the Epo gene is highly homologous in DNA sequence, and the distance between the HIF-1 and HNF-4 elements is preserved at 17-nt when the human Epo enhancer is compared with the mouse counterpart (Semenza and Wang 1992) (Fig. 2). These facts imply that the two elements may play a cooperative role in the enhancer activity of the Epo gene. So we constructed three mutant reporter plasmids which have a 6-nt deletion in the HIF-1 element (M1), two 6-nt deletions in the HNF-4 element (M2), and a 6-nt and two 6-nt deletions in both the HIF-1 and HNF-4 elements (M3), respectively, and transfected them into Hep3B cells. As shown in Fig. 1, the luciferase activity in wild-type reporter plasmid (WT) was induced 31.7-fold by hypoxia, which was comparable to the hypoxic induction of Epo in Hep 3B cells. On the other hand, M1 and M2 lost luciferase activity by $3.5 \%$ and $4.4 \%$ of the activity of wild-type reporter plasmid, respectively. They also caused loss of inducibility by almost the same degree as that in M3. These results indicate that both elements were necessary for the full function of Epo enhancer activity by hypoxia, and that synergistic up-regulation of gene expression occurred under hypoxic conditions.

Transitional change in interaction between HIF-1 and HNF-4 in response to hypoxic conditions

If HIF-1 and HNF-4 elements were necessary for the synergistic up-regulation of gene expression in hypoxia, HIF-1 and HNF-4, which bind to HIF-1 and HNF-4 elements, respectively, are, presumably, interacting with each other. To investigate possible interactions between HIF-1 and HNF-4 in both normoxia and hypoxia, we employed a mammalian two-hybrid system in vivo instead of the yeast two-hybrid system or the in-vitro protein-protein interaction method. It is reported that HIF-1 is a heterodimeric complex composed of HIF- $1 \alpha$ and ARNT, whereas HNF-4 is a homodimeric complex. So, wild-type cDNAs of HIF-1 $\alpha$, ARNT, and HNF-4 were cloned into pACT vector to fuse the VP16 activation domain, and into pBIND vector to fuse the GAL4 DNA binding domain.

These effector plasmids were transiently cotransfected into Hep3B cells along with the pG5luc expression vector and cultured in normoxia or hypoxia for $24 \mathrm{~h}$. As shown in Table 1, essentially no transcriptional activation of the luciferase gene in vivo was seen on cotransfection of either GAL4-HNF-4 and VP16-HIF-1 $\alpha$ or GAL4-HIF-1 $\alpha$ and VP16-HNF-4 in normoxia. But cotransfection of either GAL4-ARNT and VP16-HNF-4 or GAL4-HNF-4 and VP16-ARNT produced strong augmentation of the luciferase activity in normoxia. On the other hand, essentially 
Table 1. Interactions of HNF-4 with either HNF-1 $\alpha$ or ARNT under normoxic and hypoxic conditions in Hep 3B cells, using a mammalian two-hybrid assay in vivo

\begin{tabular}{lcc}
\hline & $\begin{array}{l}\text { Normoxia } \\
\text { luciferase } \\
\text { activity }\left(\times 10^{3}\right)\end{array}$ & \multicolumn{1}{c}{ Hypoxia } \\
\hline Gal4 + VP16 & $6.4 \pm 1.0$ & $12.7 \pm 2.0$ \\
GAL4-HNF-4 + VP16 & $12.7 \pm 1.6$ & $29.3 \pm 3.5$ \\
GAL4-HIF-1 $\alpha$ + VP16 & $36.8 \pm 1.4$ & $105.8 \pm 3.6$ \\
GAL4-ARNT + VP16 & $470.2 \pm 5.0$ & $580.5 \pm 52.2$ \\
GAL4 + VP16-HNF-4 & $14.8 \pm 1.3$ & $14.3 \pm 1.1$ \\
GAL4 + VP16-HIF-1 $\alpha$ & $9.4 \pm 1.9$ & $5.4 \pm 0.3$ \\
GAL4 + VP16-ARNT & $22.8 \pm 6.8$ & $24.9 \pm 2.2$ \\
GAL4-HNF-4 + VP16-HIF-1 $\alpha$ & $20.6 \pm 1.8$ & $69.4 \pm 6.6$ \\
GAL4-HNF-4 + VP16-ARNT & $150.2 \pm 19.6$ & $40.3 \pm 6.3$ \\
GAL4-HIF-1 $\alpha$ + VP16-HNF-4 & $25.7 \pm 2.4$ & $342.8 \pm 3.8$ \\
GAL4-ARNT + VP16-HNF-4 & $2148 \pm 145.7$ & $2065 \pm 61.3$ \\
\end{tabular}

Effector plasmids were constructed by clonings of wild-type cDNAs of HNF-4, HIF-1 $\alpha$ and ARNT, into either pBIND to fuse the GAL4 DNA binding domain, or pACT to fuse the VP16 transactivation domain. Hep 3B cells were cotransfected with $0.4 \mu \mathrm{g}$ each of either GAL4-HNF-4 and VP16-ARNT or VP16-HIF-1 $\alpha$, or of VP16-HNF-4 and GAL4-ARNT or GAL4-HIF-1 $\alpha$ along with $0.4 \mu \mathrm{g}$ pG5luc luciferase reporter plasmid containing five GAL4 DNA binding sites, and cultured in normoxia or hypoxia for $24 \mathrm{~h}$.

The luciferase activity was measured as described in "Materials and methods." Each value represents the means \pm SD of three independent experiments

See text for abbreviations

no activation of luciferase activity was observed upon cotransfection of GAL4-HNF-4 and VP16-ARNT in hypoxia. However, cotransfection of GAL4-HNF-4 and VP16-HIF-1 $\alpha$, or vice versa, produced a significant augmentation of luciferase activity in hypoxia. Essentially the same results were obtained with 239 cells in the same kind of experiment (data not shown). These results indicate that a transitional change probably took place from the interaction between ARNT and HNF-4 to that between HIF-1 $\alpha$ and HNF-4 in response to hypoxic conditions.

Identification of a domain involved in the interaction between ARNT and HNF-4 in normoxia

To define the region of interaction between ARNT and HNF-4 in normoxia, we constructed series of dele-tion mutants of ARNT and HNF-4 that were cloned into pACT vector and pBIND vector, respectively, and we cotransfected either wild-type GAL4-HNF-4 and various mutant VP16ARNTs or various mutant GAL4-HNF-4s and wild-type VP16-ARNT into Hep 3B cells. As shown in Fig. 3A, cotransfection of either mutant GAL4-HNF-4 (amino acids 369-465) or mutant GAL4-HNF-4 (amino acids 135-465) and wild-type VP16-ARNT produced almost the same luciferase activity as cotransfection of wild-type GAL4-HNF-4 and wild-type VP16-ARNT in normoxia. But no activation was observed on the cotransfection of mutant GAL4-HNF-4 (amino acids 1-134) containing the $\mathrm{Zn}$ finger domain and wild-type VP16-Arnt. Cotransfection of mutant GAL4HNF-4 (amino acids 135-368) containing ligand binding and dimerization domains and wild-type VP16-ARNT yielded moderate luciferase activity. These results indicate that the C-terminal sequence (amino acids 369-465) of HNF-4 was a minimal domain of interaction with ARNT.

Figure 3B shows interactions between wild-type HNF-4 and deletion mutants of ARNT in normoxia. Cotransfection of either mutant VP16-ARNT (amino acids 142-458) containing the PAS domain or mutant VP16ARNT (amino acids 1-458) and wild-type GAL4-HNF-4 in normoxia caused a significant increase in luciferase activity, but was less active than the cotransfection of wild-type GAL4-HNF-4 and wild-type VP16-ARNT. Essentially, no activation was seen on the cotransfection of any combinations between wild-type GAL4-HNF-4 and the other mutants of VP16-ARNT. These results indicate that the bHLH and PAS domains of ARNT were essential for the interaction with HNF-4.

Identification of domain involved in interaction between HIF- $1 \alpha$ and HNF-4 in hypoxia

Next we investigated which domains of HNF-4 and HIF-1 $\alpha$ were involved in the transitional change of interaction between the two transcription factors in response to hypoxia. A series of deletion mutants of HNF-4 and HIF-1 $\alpha$ were constructed and cotransfected with wild-type counterparts into Hep 3B cells. The interaction between HNF-4 and HIF-1 $\alpha$ in hypoxia appeared to be weaker than that between HNF-4 and ARNT in normoxia, probably due to a dilution effect, by an increase in the HIF-1 $\alpha$ pool in response to hypoxic conditions (Kallio et al. 1997). As shown in Fig. 4A, no activation was seen on cotransfection of mutant GAL4-HNF-4 (amino acids 1-368) and wild-type VP16-HIF-1 $\alpha$, whereas cotransfection of either mutant GAL4-HNF-4 (amino acids 1-134) containing the Zn finger domain or mutant GAL4-HNF-4 (amino acids 135-368) and wild-type VP16-HIF-1 $\alpha$ gave a slight elevation of luciferase activity in hypoxia. In contrast, cotransfection of mutant GAL4-HNF-4 (amino acids 369-465) and wild-type VP16-HIF-1 $\alpha$ yielded $57 \%$ of the luciferase activity in the wild type, while cotransfection of mutant GAL4-HNF-4 (amino acids 135-465) and wild-type VP16-HIF-1 $\alpha$ produced the same luciferase activity as cotransfection of both wild-type transcription factors. These results indicate that an extended sequence from the C-terminal of HNF-4 was necessary for the interaction with HIF-1 $\alpha$ in hypoxia.

As shown in Fig. 4B, no activation was seen on cotransfection of either mutant VP16-HIF-1 $\alpha$ (amino acids 1-105) containing the bHLH domain or mutant VP16-HIF$1 \alpha$ (amino acids 527-826) containing the C-terminal and wild-type GAL4-HNF-4 in hypoxia. However, cotransfection of either mutant VP16-HIF-1 $\alpha$ (amino acids 106-526) containing the PAS domain, or VP16-mutant HIF-1 $\alpha$ (amino acids 1-526), or VP16-mutant HIF-1 $\alpha$ (amino acids 106-826) and wild-type GAL4-HNF-4 produced similar high luciferase activity, although the activity was less than in the wild-type cotransfection. These results indicate that the PAS domain of HIF- $1 \alpha$ was a minimal domain of interaction with HNF-4 in hypoxia. 


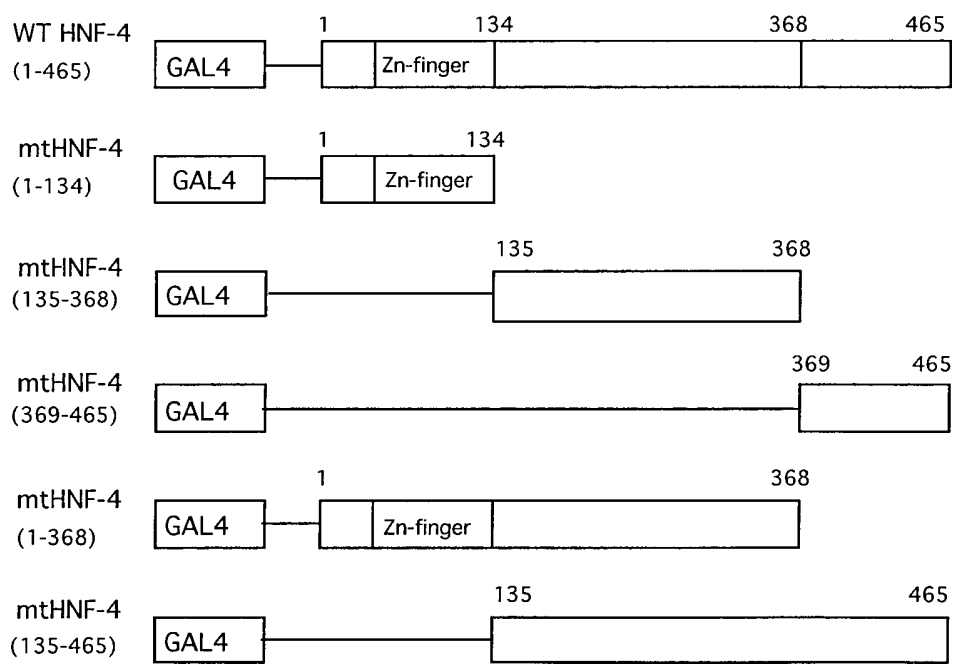

$500.0 \pm 8.5$

$11.9 \pm 1.0$

$190.9 \pm 2.1$

$425.2 \pm 5.9$

$139.7 \pm 3.1$

B

luciferase activity

$\left(x 10^{3}\right)$

VP 16

$39.9 \pm 3.6$

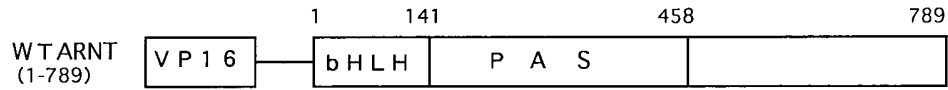

$495.8 \pm 13.4$

\begin{tabular}{lll}
$\underset{(1-141)}{\operatorname{mtARNT}} \mathrm{VP} 16$ & 141 \\
\hline & & 1 \\
& &
\end{tabular}

mtARNT

$(142-458)$

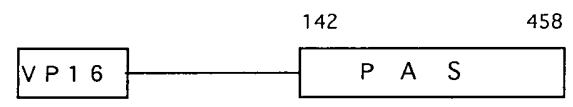

$231.1 \pm 17.3$

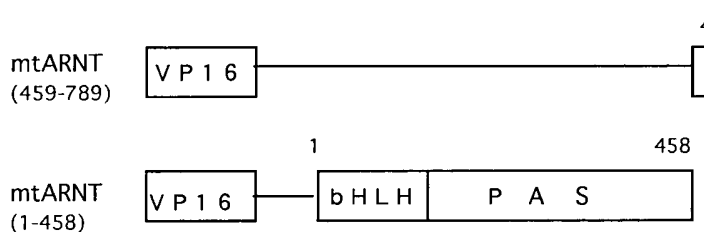

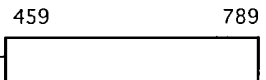

789

\author{
$(0.0 \pm 2.8$
}

$324.1 \pm 0.6$

\title{
mIARNT
}

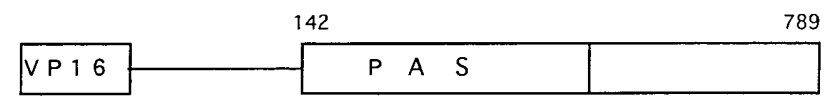

$69.0 \pm 4.4$

Fig. 3A, B. Identification of interaction domain between HNF-4 and ARNT under normoxic conditions. A Cotransfections of VP16-ARNT and various mutant GAL4-HNF-4s. Mutant GAL4-HNF-4s, amino acids 1-134 containing the $\mathrm{Zn}$ finger domain, amino acids $135-368$ containing ligand binding and dimerization domains, amino acids 369 465 containing the $\mathrm{C}$-terminal, amino acids $1-368$, and amino acids 135-465 were cloned into pBIND. Cotransfections and measurements of luciferase activity were carried out as described in "Materials and methods." Each value represents the activities on cotransfections of VP16-ARNT and various mutant GAL4-HNF-4s minus those of VP16 and the counterparts of various mutant GAL4-HNF-4s expressed as the means \pm SD of three independent experiments. B Cotransfections of GAL4-HNF-4 and various mutant VP16-ARNTs. Mutant VP16ARNTs, amino acids 1-141 containing the bHLH domain, amino acids $142-458$ containing the PAS domain, amino acids 459-789 containing the C-terminal, amino acids $1-458$, and amino acids 142-789 were cloned into pACT. Cotransfections and measurements of luciferase activity were carried out as described in "Materials and methods." Each value represents the activities on cotransfections of various mutant VP16-ARNTs and GAL4-HNF-4 minus those of the counterparts of various mutant VP16-ARNTs and GAL4 expressed as the means \pm $\mathrm{SD}$ of three independent experiments 
Fig. 4A, B. Identification of interaction domain between HNF4 and HIF-1 $\alpha$ under hypoxic conditions. A Cotransfections of VP16-HIF-1 $\alpha$ and various mutant GAL4-HNF-4s. The mutant GAL4-HNF-4s used were the same as those shown in Fig. $3 \mathrm{~A}$. B Cotransfections of GAL4HNF-4 and various mutant VP16-HIF-1as. Mutant VP16HIF-1 $\alpha$ s, amino acids 1-105 containing the bHLH domain, amino acids 106-526 containing the PAS domain, amino acids 527826 containing the C-terminal, amino acids $1-526$, and amino acids 106-826 were cloned into pACT. Cotransfections and measurements of luciferase activities were performed as described in "Materials and methods." Each value was expressed as shown in Fig. 3 luciferase activity

$\left(\times 10^{3}\right)$

GAL4

$4.0 \pm 0.4$

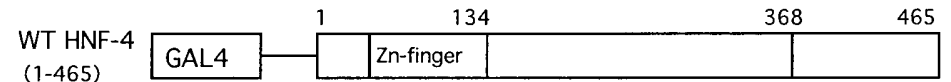

$51.6 \pm 1.9$

mtHNF-4

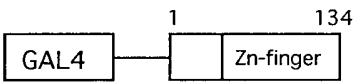

mtHNF-4

(135-368)

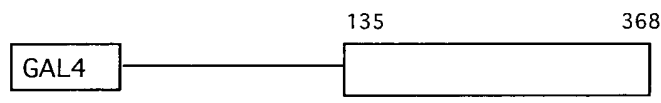

$14.4 \pm 1.2$

mtHNF-4

(369-465)

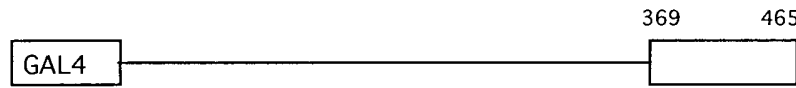

$31.2 \pm 3.0$

$\mathrm{mtHNF}-4$

(1-368)

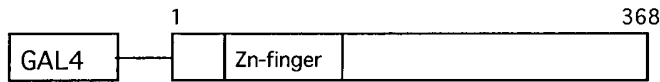

368

mtHNF-4

(135-465)

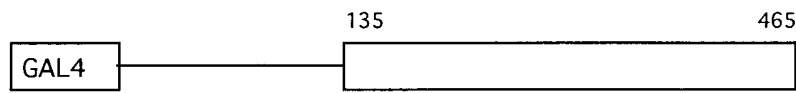

$1.0 \pm 0.2$

$46.7 \pm 1.4$

B

luciferase activity

$\left(\times 10^{3}\right)$

VP 16

$5.1 \pm 0.8$

\begin{tabular}{ll|l|lll|l|} 
WTHF-1 & \multicolumn{1}{c}{105} & \multicolumn{1}{c}{526} \\
$(1-826)$ & V P 16 & b H L H & P A & S & \\
\hline
\end{tabular}

$48.8 \pm 2.9$

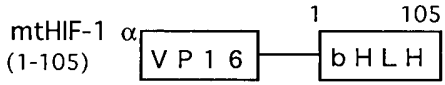

$1.0 \pm 0.3$

mtHIF-1 $\alpha$ (106-526) $\mathrm{VP} 16$

$32.8 \pm 4.0$

${ }_{(527-826)}^{\mathrm{mtHIF}-1} \alpha$

$0.4 \pm 0.06$

\begin{tabular}{ll|l|l|l|l|l|} 
mtHIF-1 & $\alpha$ \\
(1-526) & VP 16 & P A A S \\
\hline
\end{tabular}

$26.5 \pm 0.7$

${ }_{(106-826)}^{m+H I F-1} \alpha \operatorname{VP} 16$

106 826 P A S

\section{Discussion}

Our present studies of mutant reporter plasmids have demonstrated that both HIF-1 and HNF-4 elements were required for the full induction of gene expression by the Epo enhancer in response to hypoxia. This cooperative up-regulation may be achieved by transitional change from the interaction of HNF-4 with ARNT in normoxia to that with
HIF-1 $\alpha$ in hypoxia. The experiments on mutant effector plasmids revealed that domains involved in the interaction between HNF-4 and ARNT in normoxia were identified in the C-terminal amino acids 369-465, and in the N-terminal amino acids $1-458$ containing both the bHLH and PAS domains, respectively. Also, the PAS domain of HIF-1 $\alpha$ (amino acids 106-526) and the extended sequence from the C-terminal of HNF-4 (amino acids 135-465) were necessary for the interaction between the two transcription factors in hypoxia. 
Two mechanisms are reported for oxygen sensing. One mechanism is that a heme protein (Goldberg et al. 1988) senses a low level of oxygen tension, and a signal is transmitted to target genes through an as yet unidentified transduction pathway. The other is that oxygen sensor is a non-heme iron-binding protein, HIF-1 $\alpha$ itself (Srinivas et al. 1998). Whichever mechanism works, the low oxygen signal is considered to be transduced to HIF-1 or HNF-4, possibly by ligands or phosphorylation. Although, recently, fatty acyl-Co A thioesters were identified as ligands of HNF- $4 \alpha$ (Herz et al. 1998), it remains unknown what triggers the activation of HNF-4 in response to hypoxia. The mammalian two-hybrid experiments using deletion mutant effector plasmids, however, indicate that almost the same C-terminal sequence of HNF-4 was used for the interactions with both ARNT in normoxia and HIF- $1 \alpha$ in hypoxia. If HNF-4 failed to interact with HIF-1 due to deletion of the C-terminal sequence, it would lead to loss of the enhancer activity. Interestingly, Galson et al. (1995) reported that the C-terminal of HNF-4 was required for the enhancement of hypoxic induction.

On the other hand, it is more plausible that HIF-1 interacted with HNF-4 indirectly through a whole dimer that had been formed, rather than directly through each monomer, because at least the PAS domains of HIF-1, which have been implicated in dimerization and ligand binding, were necessary for the interaction with HNF-4 in both normoxia and hypoxia. So the ARNT formed dimerization with HIF$1 \alpha$ may interact with the C-terminal sequence of HNF-4 in normoxia. Alternatively, it is possible that ARNT as a homodimeric complex may interact with HNF-4, since the homodimer of ARNT is a constitutively active transcriptional regulator on symmetric $\mathrm{E}$ box motifs (Antonsson et al. 1995) and HIF-1 $\alpha$ protein is barely detectable in normoxia (Kallio et al. 1997). This interaction in normoxia may lead to a functionally repressed state of gene expression by the Epo enhancer. Once the low oxygen signal is received in response to hypoxia, HIF- $1 \alpha$ is accumulated by rendering a conformational change of HIF- $1 \alpha$ more resistant to proteolysis. At the same time, the interaction of HNF-4 with ARNT in normoxia would be shifted to that with accumulated HIF-1 $\alpha$ in hypoxia. This transitional change of the interaction may produce a conformational change in the HIF-1 and HNF-4 complex. Although it remains to be seen whether this conformational change is transduced to other functional domains of HIF-1 or HNF-4, it may allosterically modulate transcriptional regulatory functions of the Epo enhancer, and possibly this allosteric effect, in turn, may modulate the interaction of HIF-1 with transcriptional coactivators. Indeed, such coactivators as $\mathrm{CBP} / \mathrm{p} 300$ are reported to interact with the C-terminal sequence of HIF-1 $\alpha$ and to be critical for the hypoxic induction of Epo enhancer activity (Arany et al. 1996; Ebert and Bunn 1998), probably providing circumstances that strengthen the interaction between the coactivators and the basal transcriptional machinary.

Acknowledgments We thank Dr. Milind Mahajan for critical reading of the manuscript.

\section{References}

Arany Z, Huang LE, Eckner R, Bhattacharya S, Jiang C, Goldberg MA, Bunn HF, Livingston DM (1996) An essential role for p300/ CBP In the cellular response to hypoxia. Proc Natl Acad Sci USA 93:12969-12973

Antonsson C, Arulampalam V, Whitelaw ML, Pettersson S, Poellinger L (1995) Constitutive function of the basic helix-loop-helix/PAS factor Arnt. J Biol Chem 270:13968-13972

Beru N, McDonald J, Lacombe C, Goldwasser E (1986) Expression of the erythropoietin gene. Mol Cell Biol 6:2571-2575

Blanchard KL, Acquaviva AM, Galson DL, Bunn HF (1992) Hypoxia induction of the human erythropoietin gene: cooperation between the promoter and enhancer, each of which contains steroid receptor response elements. Mol Cell Biol 12:5373-5385

Bondurant MC, Koury MJ (1986) Anemia induces accumulation of erythropoietin mRNA in the kidney and liver. Mol Cell Biol 6:27312733

Ebert BL, Bunn HF (1998) Regulation of transcription by hypoxia requires a multiprotein complex that includes hypoxia-inducible factor 1 , an adjacent transcription factor, and p300/CREB binding protein. Mol Cell Biol 18:4089-4096

Ebert BL, Firth JD, Ratcliffe PJ (1995) Hypoxia and mitochondrial inhibitors regulate expression of glucose transporter-1 via distinct Cis-acting sequences. J Biol Chem 270:29083-29089

Galson DL, Tsuchiya T, Tendler DS, Huang LE, Ren Y, Ogura T, Bunn HF (1995) The orphan receptor hepatic nuclear factor 4 functions as a transcriptional activator for tissue-specific and hypoxiaspecific erythropoietin gene expression and is antagonized by EAR3/ COUP-TF1. Mol Cell Biol 15:2135-2144

Goldberg MA, Dunning SP, Bunn HF (1988) Regulation of the erythropoietin gene: evidence that the oxygen sensor is a heme protein. Science 242:1412-1415

Goldberg MA, Glass GA, Cunningham JM, Bunn HF (1987) The regulated expression of erythropoietin by two human hepatoma cell lines. Proc Natl Acad Sci USA 84:7972-7976

Herz R, Magenheim J, Berman I, Bar-Tana J (1998) Fatty acyl-CoA thioesters are ligands of hepatic nuclear factor- $4 \alpha$. Nature 392:512516

Kallio PJ, Pongratz I, Gradin K, McGuire J, Poellinger L (1997) Activation of hypoxia-inducible factor $1 \alpha$ : posttranscriptional regulation and conformational change by recruitment of the Arnt transcription factor. Proc Natl Acad Sci USA 94:5667-5672

Koury MJ, Bondurant MC (1990) Erythropoietin retards DNA breakdown and prevents programmed death in erythroid progenitor cells. Science 248:378-381

Levy AP, Levy NS, Wegner S, Goldberg MA (1995) Transcriptional regulation of the rat vascular endothelial growth factor gene by hypoxia. J Biol Chem 270:13333-13340

Miyake T, Kung CK-H, Goldwasser E (1977) Purification of human erythropoietin. J Biol Chem 252:5558-5564

Salceda S, Caro J (1997) Hypoxia-inducible factor 1 alpha protein is rapidly degraded by the ubiquitin-proteasome system under normoxia condition. J Biol Chem 272:22642-22647

Schuster SJ, Badiavas EV, Costa-Giomi P, Weinmann R, Erslev AJ, Caro J (1989) Stimulation of erythropoietin gene transcription during hypoxia and cobalt exposure. Blood 73:13-16

Semenza GL, Roth PH, Fang HM, Wang GL (1994) Transcriptional regulation of gene encoding glycolytic enzymes by hypoxia-inducible factor 1. J Biol Chem 269:23757-23763

Semenza GL, Wang GL (1992) A nuclear factor induced by hypoxia via de novo protein synthesis binds to the human erythropoietin gene enhancer at a site required for transcriptional activation. Mol Cell Biol 12:5447-5454

Srinivas V, Zhu X, Salceda S, Nakamura R, Caro J (1998) Hypoxiainducible factor $1 \alpha$ (HIF- $1 \alpha)$ is a non-heme iron protein. J Biol Chem 273:18019-18022

Wang GL, Jiang B-H, Rue EA, Semenza GL (1995) Hypoxia-inducible factor 1 is a basic-helix-loop-helix-PAS heterodimer regulated by cellular $\mathrm{O}_{2}$ tension. Proc Natl Acad Sci USA 92:5510-5514

Wang GL, Semenza GL (1995) Purification and characterization of hypoxia-inducible factor 1 . J Biol Chem 270:1230-1237 